\title{
A Novel Phase Locked Loop for Grid-Connected Converters under Non-Ideal Grid Conditions
}

\author{
Long-Yue Yang*, Chong-Lin Wang ${ }^{*}$ Jian-Hua Liu ${ }^{\dagger}$, and Chen-Xi Jia** \\ ${ }^{*}$ School of Information and Electrical Engineering, China University of Mining and Technology, Xuzhou, China \\ ${ }^{* *}$ School of Electrical Engineering and Automation, Jiangsu Normal University, Xuzhou, China
}

\begin{abstract}
Grid synchronization is one of the key techniques for the grid-connected power converters used in distributed power generation systems. In order to achieve fast and accurate grid synchronization, a new phase locked loop (PLL) is proposed on the basis of the complex filter matrixes (CFM) orthogonal signal generator (OSG) crossing-decoupling method. By combining first-order complex filters with relation matrixes of positive and negative sequence voltage components, the OSG is designed to extract specific frequency orthogonal signals. Then, the OSG mathematical model is built in the frequency-domain and time-domain to analyze the spectral characteristics. Moreover, a crossing-decoupling method is suggested to decouple the fundamental voltage. From the eigenvalue analysis point of view, the stability and dynamic performance of the new PLL method is evaluated. Meanwhile, the digital implementation method is also provided. Finally, the effectiveness of the proposed method is verified by experiments under unbalanced and distorted grid voltage conditions.
\end{abstract}

Key words: Frequency estimation, Orthogonal Signal Generator (OSG), Phase Locked Loop (PLL), Synchronization

\section{INTRODUCTION}

With the rapid development of the distributed generation technology for solar, wind and other renewable energy sources, the three-phase grid-connected inverter has been widely used in distributed generation systems. In order to connect large-scale renewable energy sources to the grid, a series of technical problems need to be solved. Among them, the grid synchronization phase locked loop is one of the basic technical requirements of distributed grid-connected generation systems [1]-[3].

Various synchronization techniques have been proposed in recent years. The typical methods, such as zero-crossing detection based PLLs [4], [5], Kalman filtering based PLLs [6], [7] and digital Fourier transform based PLLs [8], [9], can be classified as conventional PLLs. With the characteristics of low precision, slow response and sensitivity to non-ideal grid

Manuscript received Jul. 31, 2014; accepted Oct. 9, 2014

Recommended for publication by Associate Editor Sung-Yeul Park.

†Corresponding Author: liujhcumt@163.com

Tel: +86-13382679966, China University of Mining and Technology

*School of Information and Electrical Engineering, China University of

Mining and Technology, China

${ }^{* *}$ School of Electrical Engineering and Automation, Jiangsu Normal University, China environments, they are rarely used now in actual systems. Therefore, the closed-loop PLL is the current mainstream technique. By adjusting the phase difference between the input signal and the PLL output signal to zero, closed loop system synchronization can be achieved. In this system, the phase difference between the input and the output is obtained by using a Phase Detector (PD), which is generally a multiplication process. In addition, the output of the PD is used as the input of the loop filter (LF) to generate the control signal. With this, the output phase signal can be generated by a voltage controlled oscillator (VCO). The Costas PLL [10], synchronous reference frame (SRF) PLLs [11]-[13] and other intelligent adaptive algorithm based PLLs [14]-[16] are commonly used closed-loop phase-locked methods. The main difference among these different PLLs lies in how the PD block is implemented.

The synchronous reference frame PLL is the most widely used linear closed-loop PLL. Under the condition of an ideal three-phase equilibrium voltage, the frequency, amplitude and phase of the grid voltage can be detected quickly and accurately by the SRF-PLL. However, the real grid voltage is not always ideal. Sometimes fundamental negative sequence voltage and low-frequency harmonic components occur, which leads to the power quality problems of frequency fluctuation, 
three-phase unbalance and voltage distortion. In this case, the negative sequence component and harmonic component turn into low-frequency interferences in the synchronous reference frame [17]. The PLL performance is badly affected with the occurrence of output frequency oscillations, and sometimes the PLL cannot work normally.

Different advanced synchronization systems able to deal with these grid conditions have been proposed in the literature as an improvement to the SRF-PLL. [18] and [19] use a notch filter with a specific frequency to eliminate the second harmonic on the $d-q$ reference frame. The filtering performances of these are affected by frequency variations. In [20], the positive sequence voltage component is obtained by the symmetrical component method and the corresponding matrix transformation. However, a lot of table search operations and mathematical operations are required. The dual synchronous reference frame PLL has been proposed in [21]. However, the structure is complex, and a great deal of computation is required. Among the numerous methods, some techniques to create an orthogonal signal from the original input signal are put forward. The earliest, and probably the simplest, orthogonal signal generator (OSG) is a transfer delay block [22]. This technique presents satisfactory results if the grid frequency is at its rated value. However, when the grid voltage undergoes frequency variations, the output signal of the OSG will not be exactly orthogonal. An extraction method for the fundamental positive and negative sequence components based on delay signals elimination is elaborated in [23]-[25], where an accurate delay may not be achieved by the data cache under frequency-varying conditions. In [26] and [27], a Kalman filter based OSG is proposed. However, the problems of covariance matrix selection and measurement error of the Kalman filter have a bad influence on its application. Second-order generalized integrators (SOGIs) are also used to separate the fundamental positive and negative sequence components [28], [29]. Frequency adaptive performance, low computational burden, and a relatively high filtering capability are the advantages of this method. They make it a successful solution for harmonically distorted and frequency-varying conditions. Thus, the study of SOGI and similar structures has become a research focus in recent years [30], [31].

This paper proposes a new technique for a phase locked loop under unbalanced and distorted operating conditions. The proposed orthogonal signal generator module is described as a CFM-OSG since it is based on first-order complex filters working together inside the relation matrixes of the positive and negative sequence voltage components. It is designed to extract specific frequency orthogonal signals. Moreover, a crossing-decoupling method is put forward to decouple the fundamental voltage. Then, the characteristics are analyzed, and the parameters and the discretized system are designed for the proposed CFM-OSG PLL. With a definite physical meaning, the proposed method realizes the filtering of

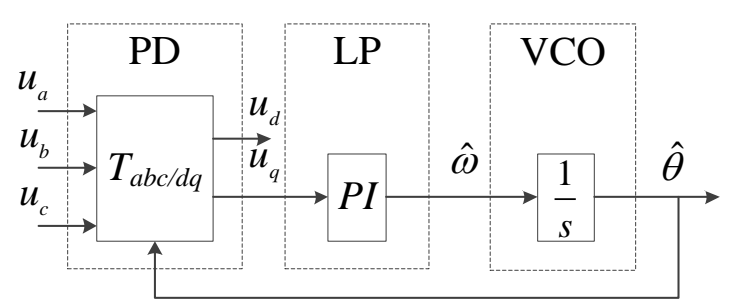

Fig. 1. Structure of SRF-PLL.

harmonic and negative sequence components, ensuring a fast transient response, a high disturbance rejection capability, and a robust performance.

The rest of this paper is organized as follows. In Section II, an orthogonal signal generator based on complex filter matrixes is proposed for combining the first-order complex filters and the relation matrixes. This is followed by an analysis of the spectral characteristics. In Section III, a crossing-decoupling method is put forward to decouple the fundamental voltage. Then, the parameters and the discretized system are designed for the proposed CFM-OSG PLL in Section IV. Section V is devoted to experiments. Finally, in Section VI, some conclusions are drawn.

\section{DESIGN OF AN OSG BASED ON COMPLEX FILTER MATRIXES}

The structure of a conventional SRF-PLL based on the synchronous reference frame is shown in Fig. 1. The park coordinate transformation is equivalent to the phase detector, and the integral link is equal to the voltage controlled oscillator. Generally, the loop filter is a proportional-integral (PI) controller.

$u_{a}, u_{b}$ and $u_{c}$ denote the three-phase voltages, respectively. $u_{d}$ and $u_{q}$ denote the $d$-axis voltage and the $q$-axis voltage in the synchronous reference frame, respectively. $\hat{\omega}$ denotes the frequency output of the PLL. $\hat{\theta}$ denotes the phase output of the PLL.

\section{A. First-order Complex Filter Theory}

In order to eliminate the influence of both harmonic voltage and fundamental negative sequence voltage, fundamental positive sequence component extraction must be completed before the calculation in the synchronous reference frame. This means that filters can be added after the Clarke transform. Therefore, the first-order complex filters are introduced in this paper as shown in Eq. (1) and Eq. (2), where Eq. (1) is a positive sequence filter and Eq. (2) is a negative sequence filter.

$$
\begin{aligned}
& F^{+}(s)=\frac{\omega_{c}}{s-j \hat{\omega}+\omega_{c}}, \\
& F^{-}(s)=\frac{\omega_{c}}{s+j \hat{\omega}+\omega_{c}},
\end{aligned}
$$

where $\hat{\omega}$ denotes the fundamental frequency output of the 


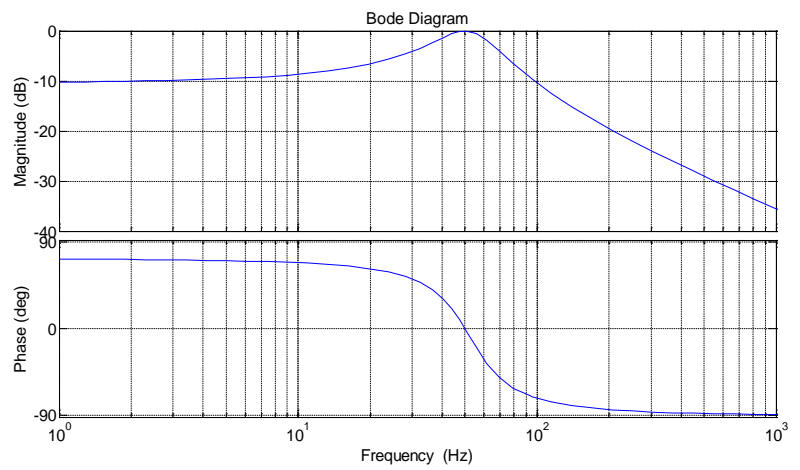

Fig. 2. Bode diagram of positive sequence filter.

PLL, and $\omega_{c}$ denotes the cut-off frequency of the filters. Set $\hat{\omega}=314(\mathrm{rad} / \mathrm{s})$, and the characteristic of the positive sequence filter is shown in Fig. 2.

After deducing the amplitude-frequency function and the phase-frequency function, the following conclusions can be obtained. 1) The amplitude of the positive sequence filter is 1 at $\hat{\omega}$, accompanied by a 0 phase shift. 2) With a signal frequency far away from $\hat{\omega}$, the signal amplitude attenuates rapidly. 3) At the frequency of $-\hat{\omega}$, the amplitude of the positive sequence filter is $\omega_{c} / \sqrt{4 \hat{\omega}^{2}+\omega_{c}^{2}}$, and the phase shift is $\arctan \left(2 \hat{\omega} / \omega_{c}\right)$. Similarly, the fundamental negative sequence voltage signal can get through the negative sequence filter with no attenuation and 0 phase shift. However, the fundamental positive sequence signal attenuates partly after getting through the filter. It is obvious that only the suppression of the harmonic component can be achieved when the two kinds of first-order complex filter used separately. However, the positive and negative sequence components are still not decoupled. Therefore, the combined use of two types of filters is adopted in this paper to separate the positive and negative sequence components.

\section{B. Design of the Complex Filter Matrixes}

In order to simplify the analysis, the harmonic components in the three-phase voltage are ignored. Then, according to Lyon's method [32], under the condition of a three-phase voltage unbalance, the fundamental positive sequence, negative sequence and zero sequence voltages can be expressed as:

$$
\begin{aligned}
& \boldsymbol{U}_{a b c}^{+}=\left[\begin{array}{l}
u_{a}^{+} \\
u_{b}^{+} \\
u_{c}^{+}
\end{array}\right]=\boldsymbol{T}^{+}\left[\begin{array}{l}
u_{a} \\
u_{b} \\
u_{c}
\end{array}\right]=\frac{1}{3}\left[\begin{array}{ccc}
1 & a & a^{2} \\
a^{2} & 1 & a \\
a & a^{2} & 1
\end{array}\right]\left[\begin{array}{l}
u_{a} \\
u_{b} \\
u_{c}
\end{array}\right] \\
& \boldsymbol{U}_{a b c}^{-}=\left[\begin{array}{l}
u_{a}^{-} \\
u_{b}^{-} \\
u_{c}^{-}
\end{array}\right]=\boldsymbol{T}^{-}\left[\begin{array}{l}
u_{a} \\
u_{b} \\
u_{c}
\end{array}\right]=\frac{1}{3}\left[\begin{array}{ccc}
1 & a^{2} & a \\
a & 1 & a^{2} \\
a^{2} & a & 1
\end{array}\right]\left[\begin{array}{l}
u_{a} \\
u_{b} \\
u_{c}
\end{array}\right], \\
& \boldsymbol{U}_{a b c}^{0}=\left[\begin{array}{l}
u_{a}^{0} \\
u_{b}^{0} \\
u_{c}^{0}
\end{array}\right]=\boldsymbol{T}^{0}\left[\begin{array}{l}
u_{a} \\
u_{b} \\
u_{c}
\end{array}\right]=\frac{1}{3}\left[\begin{array}{lll}
1 & 1 & 1 \\
1 & 1 & 1 \\
1 & 1 & 1
\end{array}\right]\left[\begin{array}{l}
u_{a} \\
u_{b} \\
u_{c}
\end{array}\right]
\end{aligned}
$$

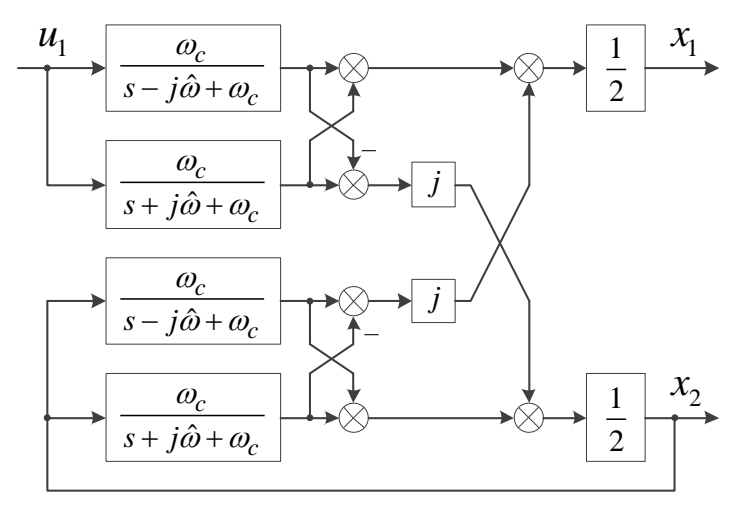

Fig. 3. The OSG model based on complex filter matrixes.

where $a=e^{j(2 \pi / 3)}$ denotes the phase-shift operator for the three-phase sinusoidal signals. After the Clarke transform, the fundamental positive and negative sequence components are coupled in $u_{\alpha}$ and $u_{\beta}$. In addition, the zero sequence voltage independently turns to $u_{\gamma}$. On the $\alpha-\beta$ reference frame, the fundamental positive and negative sequence voltage vectors can be expressed as:

$$
\begin{aligned}
\boldsymbol{U}_{\alpha \beta}^{+} & =\boldsymbol{T}_{\alpha \beta} \boldsymbol{U}_{a b c}^{+}=\boldsymbol{T}_{\alpha \beta} \boldsymbol{T}^{+} \boldsymbol{U}_{a b c}=\boldsymbol{T}_{\alpha \beta} \boldsymbol{T}^{+} \boldsymbol{T}_{\alpha \beta}^{T} \boldsymbol{U}_{\alpha \beta} \\
& =\frac{1}{2}\left[\begin{array}{cc}
1 & -q \\
q & 1
\end{array}\right]\left[\begin{array}{l}
u_{\alpha} \\
u_{\beta}
\end{array}\right] \\
\boldsymbol{U}_{\alpha \beta}^{-} & =\boldsymbol{T}_{\alpha \beta} \boldsymbol{U}_{a b c}^{-}=\boldsymbol{T}_{\alpha \beta} \boldsymbol{T}^{-} \boldsymbol{U}_{a b c}=\boldsymbol{T}_{\alpha \beta} \boldsymbol{T}^{-} \boldsymbol{T}_{\alpha \beta}^{T} \boldsymbol{U}_{\alpha \beta} \\
& =\frac{1}{2}\left[\begin{array}{cc}
1 & q \\
-q & 1
\end{array}\right]\left[\begin{array}{l}
u_{\alpha} \\
u_{\beta}
\end{array}\right]
\end{aligned}
$$

In Eq. (4) and Eq. (5), $\boldsymbol{T}_{\alpha \beta}=\sqrt{\frac{2}{3}}\left[\begin{array}{ccc}1 & -1 / 2 & -1 / 2 \\ 0 & \sqrt{3} / 2 & -\sqrt{3} / 2\end{array}\right]$ is the Clarke transform matrix. In addition, $q=e^{-j(\pi / 2)}$ denotes the phase-shift operator for the two-phase sinusoidal signals.

Based on the above recognitions, the singular matrixes $\boldsymbol{B}^{+}=\frac{1}{2}\left[\begin{array}{cc}1 & -j \\ j & 1\end{array}\right]$ and $\boldsymbol{B}^{-}=\frac{1}{2}\left[\begin{array}{cc}1 & j \\ -j & 1\end{array}\right]$ are chosen to construct the complex filter matrixes in the complex frequency domain.

$$
\begin{aligned}
& \boldsymbol{B}^{+} \cdot\left[\begin{array}{cc}
F^{+}(s) & 0 \\
0 & F^{+}(s)
\end{array}\right]=\frac{1}{2}\left[\begin{array}{cc}
\frac{\omega_{c}}{s-j \hat{\omega}+\omega_{c}} & -j \frac{\omega_{c}}{s-j \hat{\omega}+\omega_{c}} \\
j \frac{\omega_{c}}{s-j \hat{\omega}+\omega_{c}} & \frac{\omega_{c}}{s-j \hat{\omega}+\omega_{c}}
\end{array}\right], \\
& \boldsymbol{B}^{-} \cdot\left[\begin{array}{cc}
F^{-}(s) & 0 \\
0 & F^{-}(s)
\end{array}\right]=\frac{1}{2}\left[\begin{array}{cc}
\frac{\omega_{c}}{s+j \hat{\omega}+\omega_{c}} & j \frac{\omega_{c}}{s+j \hat{\omega}+\omega_{c}} \\
-j \frac{\omega_{c}}{s+j \hat{\omega}+\omega_{c}} & \frac{\omega_{c}}{s+j \hat{\omega}+\omega_{c}}
\end{array}\right] .
\end{aligned}
$$

In order to achieve the separation of the positive and negative sequence signals, the OSG signal processing module is designed on the basis of the complex filter matrixes. Define the module inputs as $u_{1}$ and $u_{2}$, and the outputs as $x_{1}$ and $x_{2}$. By creating a structure for the linear operation of the complex matrixes, the OSG module can be expressed as follows. 
$\left.\left[\begin{array}{l}x_{1}(s) \\ x_{2}(s)\end{array}\right]=\boldsymbol{B}^{+} \cdot\left(\left[\begin{array}{cc}F^{+}(s) & 0 \\ 0 & F^{+}(s)\end{array}\right]\left[\begin{array}{l}u_{1}(s) \\ u_{2}(s)\end{array}\right]\right)+\boldsymbol{B}^{-} \cdot\left(\begin{array}{cc}F^{-}(s) & 0 \\ 0 & F^{-}(s)\end{array}\right]\left[\begin{array}{l}u_{1}(s) \\ u_{2}(s)\end{array}\right]\right)$
$=\left(\boldsymbol{B}^{+}\left[\begin{array}{cc}F^{+}(s) & 0 \\ 0 & F^{+}(s)\end{array}\right]+\boldsymbol{B}^{-}\left[\begin{array}{cc}F^{-}(s) & 0 \\ 0 & F^{-}(s)\end{array}\right]\right)\left[\begin{array}{l}u_{1}(s) \\ u_{2}(s)\end{array}\right]$
$=\frac{1}{2}\left[\begin{array}{cc}\frac{\omega_{c}}{s-j \hat{\omega}+\omega_{c}}+\frac{\omega_{c}}{s+j \hat{\omega}+\omega_{c}} & -j \frac{\omega_{c}}{s-j \hat{\omega}+\omega_{c}}+j \frac{\omega_{c}}{s+j \hat{\omega}+\omega_{c}} \\ j \frac{\omega_{c}}{s-j \hat{\omega}+\omega_{c}}-j \frac{\omega_{c}}{s+j \hat{\omega}+\omega_{c}} & \frac{\omega_{c}}{s-j \hat{\omega}+\omega_{c}}+\frac{\omega_{c}}{s+j \hat{\omega}+\omega_{c}}\end{array}\right]\left[\begin{array}{l}u_{1}(s) \\ u_{2}(s)\end{array}\right]$

Set $u_{2}=x_{2}$ (i.e., $x_{2}$ is regarded as an input signal). The internal signal can be processed through the feedback. Then the OSG model based on the complex filter matrixes is shown in Fig. 3.

Through mathematical simplifications, Eq. (9) can be obtained as follows:

$$
\left[\begin{array}{c}
x_{1}(s) \\
x_{2}(s)
\end{array}\right]=\left[\begin{array}{cc}
\frac{\omega_{c}\left(s+\omega_{c}\right)}{s^{2}+2 \omega_{c} s+\omega_{c}^{2}+\hat{\omega}^{2}} & \frac{\omega_{c} \hat{\omega}}{s^{2}+2 \omega_{c} s+\omega_{c}^{2}+\hat{\omega}^{2}} \\
\frac{-\omega_{c} \hat{\omega}}{s^{2}+2 \omega_{c} s+\omega_{c}^{2}+\hat{\omega}^{2}} & \frac{\omega_{c}\left(s+\omega_{c}\right)}{s^{2}+2 \omega_{c} s+\omega_{c}^{2}+\hat{\omega}^{2}}
\end{array}\right]\left[\begin{array}{l}
u_{1}(s) \\
x_{2}(s)
\end{array}\right] .
$$

\section{Characteristics of the CFM-OSG}

It is obvious that the imaginary part of the CFM-OSG module can be eliminated through calculation and feedback. The simplified form of Eq. (9) is easy to achieve for the digital controller. From this simplified model, the transfer function of the CFM-OSG can be expressed as:

$$
\begin{aligned}
& G_{1}(s)=\frac{x_{1}(s)}{u_{1}(s)}=\frac{\omega_{c} s}{s^{2}+\omega_{c} s+\hat{\omega}^{2}}, \\
& G_{2}(s)=\frac{x_{2}(s)}{u_{1}(s)}=\frac{-\omega_{c} \hat{\omega}}{s^{2}+\omega_{c} s+\hat{\omega}^{2}} .
\end{aligned}
$$

Eq. (10) and Eq. (11) show that the system transfer function is similar to the second-order generalized integrator. To test the performance of the CFM-OSG, the frequency characteristics of the module are analyzed.

The CFM-OSG module can be described by the following differential equation:

$$
\left\{\begin{array}{l}
\ddot{x}_{2}+\omega_{c} \dot{x}_{2}+\hat{\omega}^{2} x_{2}=-\omega_{c} \hat{\omega} u_{1} \\
x_{1}=-\dot{x}_{2} / \hat{\omega}
\end{array} .\right.
$$

Assume that input signal is $u_{1}=A_{0} \cos \left(\omega_{0} t+\phi\right)$, and that angular frequency is $\omega_{0}$. As a result, the solution of differential equation (12) is:

$$
\left[\begin{array}{l}
x_{1}(t) \\
x_{2}(t)
\end{array}\right]=C\left[\begin{array}{c}
\cos \omega_{0} t \\
\sin \omega_{0} t
\end{array}\right]+\left[\begin{array}{l}
d_{1}(t) \\
d_{2}(t)
\end{array}\right],
$$

where:

$$
\begin{aligned}
& \boldsymbol{C}=\frac{A_{0} \omega_{c}}{\left(\hat{\omega}^{2}-\omega_{0}^{2}\right)^{2}+\omega_{0}^{2} \omega_{c}^{2}} \bullet \\
& {\left[\begin{array}{cc}
\omega_{0}\left[-\left(\hat{\omega}^{2}-\omega_{0}^{2}\right) \sin \phi+\omega_{c} \omega_{0} \cos \phi\right] & \omega_{0}\left[-\omega_{c} \omega_{0} \sin \phi-\left(\hat{\omega}^{2}-\omega_{0}^{2}\right) \cos \phi\right] \\
\hat{\omega}\left[-\left(\hat{\omega}^{2}-\omega_{0}^{2}\right) \cos \phi-\omega_{c} \omega_{0} \sin \phi\right] & \hat{\omega}\left[-\omega_{c} \omega_{0} \cos \phi+\left(\hat{\omega}^{2}-\omega_{0}^{2}\right) \sin \phi\right]
\end{array}\right]}
\end{aligned}
$$

$d_{1}$ and $d_{2}$ are the exponential decay components, the decay rate of which is determined by $\omega_{c}$.

Under the special condition of $\hat{\omega}=\omega_{0}$ while ignoring the

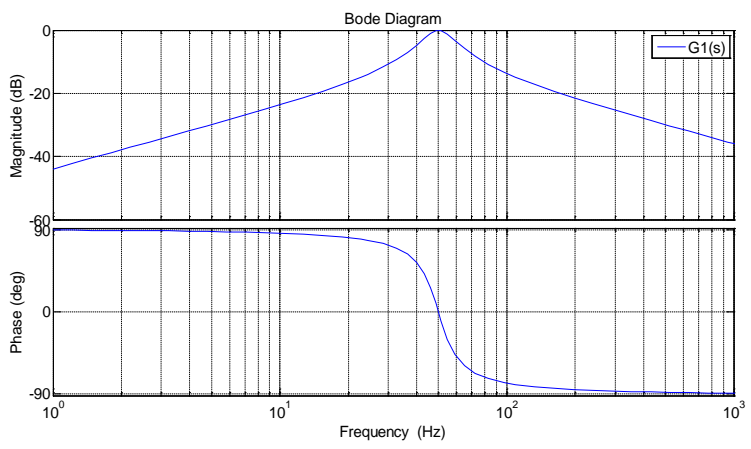

(a)

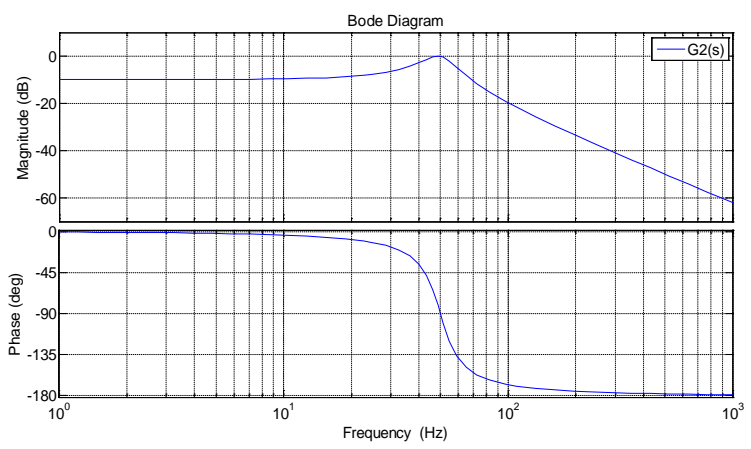

(b)

Fig. 4. Bode diagram of CFM-OSG. (a) $\mathrm{G}_{1}$ (s). (b) $\mathrm{G}_{2}$ (s).

transient terms, the solution of the system can be written as:

$$
\left\{\begin{array}{c}
x_{1}(t)=A_{0} \cos \left(\omega_{0} t+\phi\right) \\
x_{2}(t)=-A_{0} \sin \left(\omega_{0} t+\phi\right)
\end{array} .\right.
$$

From Eq. (14), it can be concluded that with a special frequency input, the CFM-OSG module outputs the original signal and its orthogonal signal after the transient process. This provides the basis for the separation of the fundamental positive sequence voltage and the negative component.

The frequency characteristics of the OSG are analyzed to observe the frequency selection and filtering ability. The spectral characteristics of the CFM-OSG are shown in Fig. 4.

It is shown in Fig. 4 that the transfer function $G_{1}$ exhibits a bandpass filtering behavior with the center frequency of $\hat{\omega}$. The amplitude attenuation and phase shift are 0 at $\hat{\omega}$ which has already been proved by (14). In addition, the signal out of the bandwidth range is strongly attenuated. If, somehow, $\hat{\omega}$ is equal to the fundamental frequency, then $x_{1}$ will match the fundamental component of the input voltage (i.e., $u_{1}$ ) in both amplitude and phase. In addition, the bandwidth is determined by the filter cut-off frequency $\omega_{c}$. A lower $\omega_{c}$ leads to a narrower bandwidth with better filtering capability, slower dynamic response speed and weaker disturbance rejection capability, and vice versa. Therefore, the filter parameter should be selected by making a compromise to satisfy the system requirements. The transfer function $G_{2}$ presents a low-pass filtering characteristic. Moreover, if $\hat{\omega}$ is equal to the fundamental voltage frequency, then $x_{2}$ will match in 


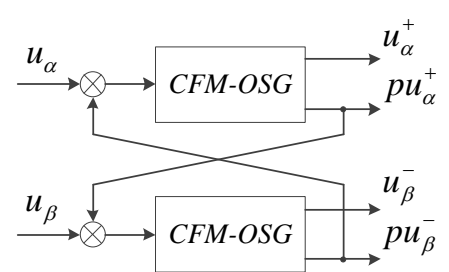

Fig. 5. Crossing feedback decoupling method based on CFM-OSG.

amplitude but with a -90 ॰ difference with the fundamental component of the input voltage $u_{1}$.

When compared with a first-order complex filter, the CFM-OSG module can filter the harmonic component in the input signal and realize the orthogonal signal extraction. Therefore, the fundamental positive and negative sequence voltages can be decoupled by taking advantage of the difference in their orthogonal signals.

\section{DESIGN OF A PLL BASED ON CFM-OSG}

A crossing feedback method is presented to decouple the fundamental voltage. By sending orthogonal signals of fundamental voltage to the input, the positive and negative sequence components can be separated into $\alpha$-axis and $\beta$-axis components, respectively. This method is shown in Fig. 5, where $p=e^{j(\pi / 2)}$ denotes the phase-shift operator for two-phase sinusoidal signals.

Combining Eq. (10) and Eq. (11), the relationship between the input and output can be expressed as:

$$
\left\{\begin{array}{l}
u_{\alpha}^{+}(s)=G_{1}(s)\left[u_{\alpha}(s)-\frac{\hat{\omega}}{s} u_{\beta}^{-}(s)\right] \\
-\frac{\hat{\omega}}{s} u_{\alpha}^{+}(s)=G_{2}(s)\left[u_{\alpha}(s)-\frac{\hat{\omega}}{s} u_{\beta}^{-}(s)\right] \\
u_{\beta}^{-}(s)=G_{1}(s)\left[u_{\beta}(s)-\frac{\hat{\omega}}{s} u_{\alpha}^{+}(s)\right] \\
-\frac{\hat{\omega}}{s} u_{\beta}^{-}(s)=G_{2}(s)\left[u_{\beta}(s)-\frac{\hat{\omega}}{s} u_{\alpha}^{+}(s)\right]
\end{array} .\right.
$$

Thus:

$$
u_{\alpha}^{+}(s)=\frac{G_{1}(s) u_{\alpha}(s)+G_{1}(s) G_{2}(s) u_{\beta}(s)}{1+\frac{\hat{\omega}}{s} G_{1}(s) G_{2}(s)} .
$$

The fundamental positive sequence voltage can be calculated on the $\alpha-\beta$ reference frame, as shown in Eq. (17).

$$
u_{\beta}(s)=-\frac{s}{\hat{\omega}} u_{\alpha}(s) \text {. }
$$

The transfer function of the positive sequence component can be derived as:

$$
\begin{aligned}
G_{\alpha}^{+}(s) & =\frac{u_{\alpha}^{+}(s)}{u_{\alpha}(s)}=\frac{G_{1}(s)-\frac{s}{\hat{\omega}} G_{1}(s) G_{2}(s)}{1+\frac{\hat{\omega}}{s} G_{1}(s) G_{2}(s)} . \\
& =\frac{\left(s^{2}+2 \omega_{c} s+\hat{\omega}^{2}\right) \omega_{c} s}{\left(s^{2}+\omega_{c} s+\hat{\omega}^{2}\right)^{2}-\omega_{c}^{2} \hat{\omega}^{2}}
\end{aligned}
$$

Similarly, Eq. (19) can be obtained for the fundamental negative sequence voltage.

$$
u_{\beta}(s)=\frac{s}{\hat{\omega}} u_{\alpha}(s) \text {. }
$$

Then, the corresponding transfer function is:

$$
\begin{aligned}
G_{\alpha}^{-}(s) & =\frac{u_{\alpha}^{+}(s)}{u_{\alpha}(s)}=\frac{G_{1}(s)+\frac{s}{\hat{\omega}} G_{1}(s) G_{2}(s)}{1+\frac{\hat{\omega}}{s} G_{1}(s) G_{2}(s)} . \\
& =\frac{\left(s^{2}+\hat{\omega}^{2}\right) \omega_{c} s}{\left(s^{2}+\omega_{c} s+\hat{\omega}^{2}\right)^{2}-\omega_{c}^{2} \hat{\omega}^{2}}
\end{aligned}
$$

According to Eq. (18) and Eq. (20), it is easy to obtain $G_{\alpha}^{+}(j \hat{\omega})=1$ and $G_{\alpha}^{-}(j \hat{\omega})=0$. In this way, the positive sequence component is extracted on the $\alpha$-axis. Meanwhile, the negative sequence component is extracted on the $\beta$-axis. On the basis of the decoupled fundamental voltage, the positive sequence voltage can be accurately estimated for the PLL. The PLL model can be obtained as shown in Fig. 6 .

Based on the aforementioned analysis, the positive sequence voltage component can be accurately estimated by the CFM-OSG feedback decoupling method in the case of a fixed frequency. However, the real grid frequency may sometimes fluctuate or offset. In the frequency fluctuation ranges, such as the $(-0.7,0.5) \mathrm{Hz}$ that the IEEE Std. 1547 allows, the frequency and phase information can be detected quickly and accurately by the PLL. Therefore, the system works stably with this adaptive frequency adjustment which reduces the transient error of the output voltage.

\section{ANALYSIS OF THE PLL SYSTEM}

\section{A. Parameters Design}

When compared with the conventional mathematical model of the SRF-PLL, the OSG decoupling link is added to the system. Therefore, both $k p$ and $k i$ (i.e. the PI controller parameters) and $\omega_{c}$ (i.e. the complex filter parameter) need to be designed. Under transient conditions, the dynamic response depends mainly on the filter parameter $\omega_{c}$. In order to realize the dynamic characteristics of a quick response and good filtering capability, it is necessary to optimize the parameter $\omega_{c}$.

Eigenvalue analysis is one of the most commonly used methods to assess system stability and dynamic performance. Based on the stability condition, the closed-loop poles of the system are all located in the left half side of the Laplace plane. This means that the real parts of the eigenvalues selected are negative.

Generally, the system transient characteristics are mainly affected by the eigenvalues. The system step response attenuates fast when the distance between the eigenvalue and the imaginary axis is long, and vice versa. Based on Eq. (18) and Eq. (20), for a given frequency $\hat{\omega}=314(\mathrm{rad} / \mathrm{s})$, the eigenvalue distribution is shown in Fig. 7 with different values of $\omega_{c}$. Notice that when the value of $\omega_{c}$ increases from 0 to 260 , 


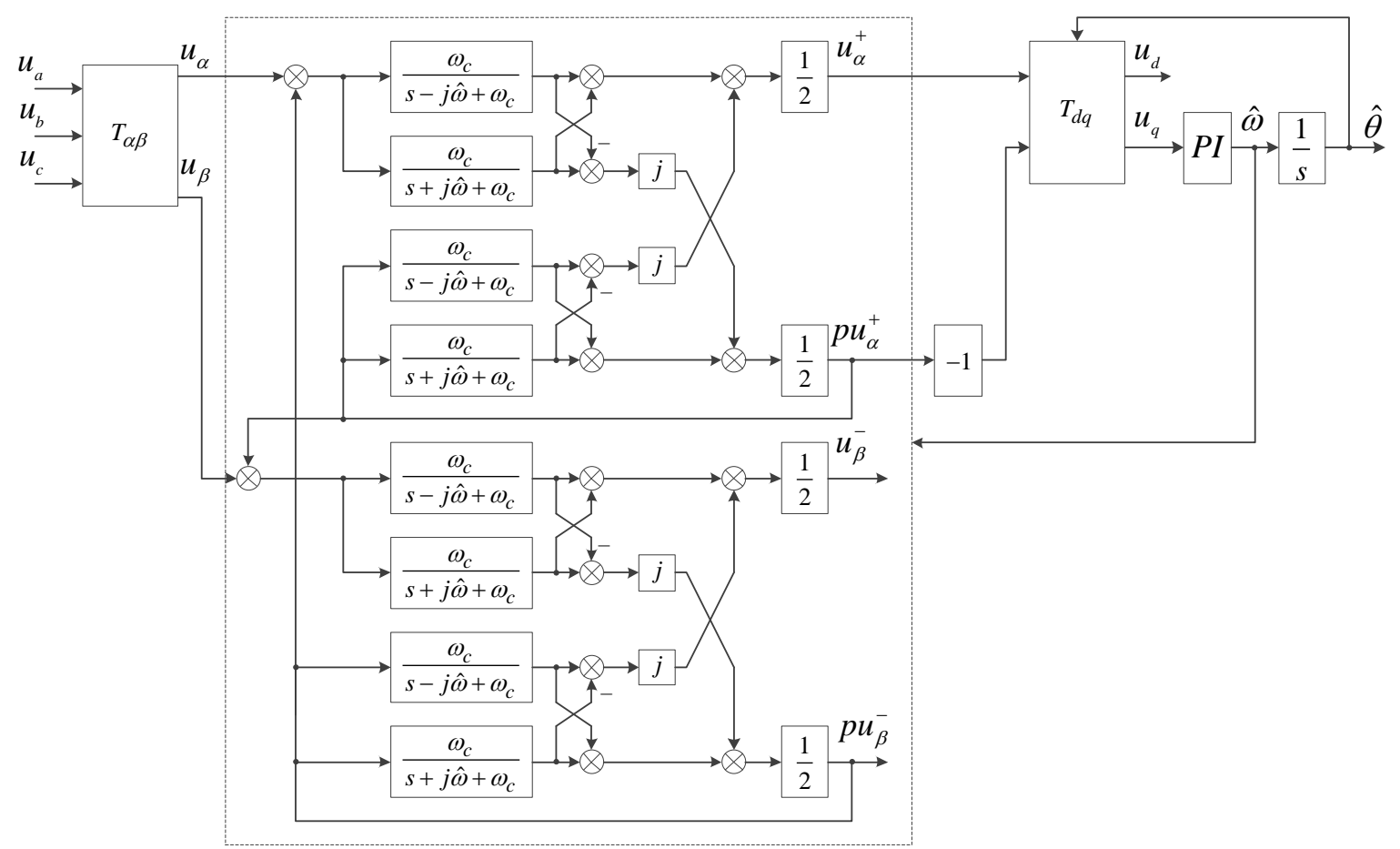

Fig. 6. PLL based on CFM-OSG Feedback decoupling method.

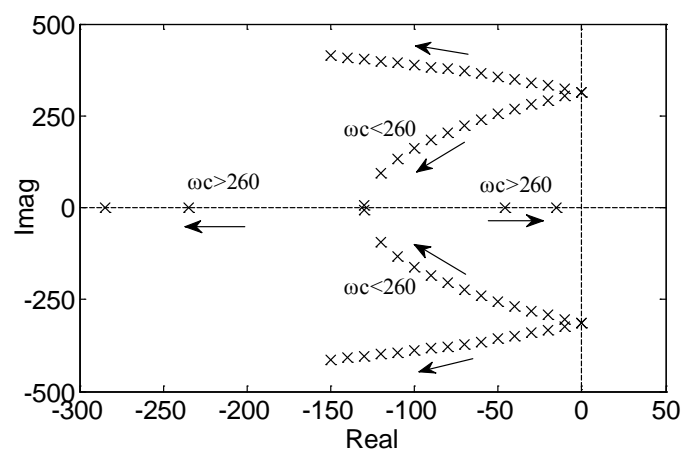

Fig. 7. Eigenvalues distribution.

the dominant eigenvalues move away from the imaginary axis, accelerating the system response speed. The opposite case occurs when the dominant eigenvalues move closer to the imaginary axis, with the value of $\omega_{c}$ increasing from 260 to 314. Consequently, in consideration of the filtering effect and the dynamic performance, the parameter is selected as $\omega_{c}=(2 \sqrt{2}-2) \hat{\omega}=260(\mathrm{rad} / \mathrm{s})$.

In this case, the spectral characteristic of the decoupling module is graphically shown in Fig. 8. For the positive sequence component, the decoupling module exhibits a bandpass filtering characteristic. But for the negative sequence component, it presents a notch filtering behavior with a center frequency of $\hat{\omega}$.

Therefore, for the PLL, the decoupling module achieves fundamental negative sequence voltage filtering and harmonic component attenuation in the $\alpha-\beta$ reference frame. When it is

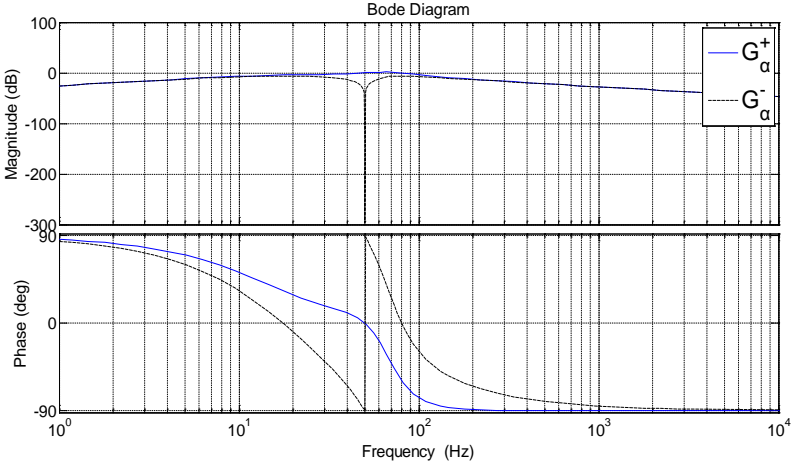

Fig. 8. Bode diagram of decoupling module.

transformed to the synchronous reference frame, the module filters the second harmonic and other harmonics. In other words, for a fundamental voltage of $50 \mathrm{~Hz}$, the cut-off frequency of the transfer function is less than $100 \mathrm{~Hz}$ in the $d-q$ reference frame. Hence, the PI controller parameters designing is similar to that in the conventional SRF-PLL.

The transfer function of the conventional SRF-PLL can be expressed as:

$$
H(s)=\frac{U k_{p} s+U k_{i}}{s^{2}+U k_{p} s+U k_{i}},
$$

where $U$ denotes the amplitude of the synchronous rotating voltage vector. The PLL is a reference tracking system, where the PI controller input is the angle error, and the output is the frequency. In the PI controller, a $k p$ with a large value leads to a faster system response speed which weakens the stability of the system, and vice versa. Considering that grid frequency 
fluctuation is small under normal circumstances, a small value is usually set to the tracking speed to ensure system stability. Based on the experience parameters and the relationship between the input and output of the controller, the restriction of the controller output in a single sampling period is:

$$
\Delta \hat{\omega} / 100<U \Delta \hat{\omega} T_{s}\left(k_{p}+k_{i}\right)<\Delta \hat{\omega},
$$

where $T s$ denotes the sampling period. The corresponding closed-loop system is a second-order overdamping system with a zero. In addition, the damping coefficient of the system can be expressed as:

$$
\xi=\frac{U k_{p}}{2 \sqrt{U k_{i}}}>1 .
$$

In addition, the filter and PD have an impact on the system response. Since the filter is a high-order system, the analysis is difficult. A computer simulation is adopted to design the PI controller parameters, which is restricted by Eq. (22) and Eq. (23). By comparing the fluctuations and speeds of the dynamic response, the optimal controller parameters are selected as $k p=0.7$ and $k i=2$. With these parameters, a small output frequency fluctuation, fast tracking speed and strong robustness can be achieved when the system parameters change.

\section{B. Digital Implementation of the PLL}

Generally, the z-transform is used to discretize the control algorithm for a digital controller. Since the main components of the grid voltage signal are fundamental and low order harmonics, the Tustin transform is appropriate in that it may keep a good linear relationship between the s-domain and the z-domain in the low frequency band. To make calculations without imaginary terms, Eq. (9) is finally selected to be discretized.

The Tustin transform equation can be expressed as:

$$
s=\frac{2}{T_{s}} \frac{z-1}{z+1} .
$$

Substituting Eq. (24) into Eq. (9) yields:

$$
\left[\begin{array}{c}
x_{1}(z) \\
x_{2}(z)
\end{array}\right]=\left[\begin{array}{cc}
\frac{b_{2} z^{-2}+b_{1} z^{-1}+b_{0} z}{a_{2} z^{-2}+a_{1} z^{-1}+a_{0} z} & \frac{b_{5} z^{-2}+b_{4} z^{-1}+b_{3} z}{a_{2} z^{-2}+a_{1} z^{-1}+a_{0} z} \\
-\frac{b_{5} z^{-2}+b_{4} z^{-1}+b_{3} z}{a_{2} z^{-2}+a_{1} z^{-1}+a_{0} z} & \frac{b_{2} z^{-2}+b_{1} z^{-1}+b_{0} z}{a_{2} z^{-2}+a_{1} z^{-1}+a_{0} z}
\end{array}\right]\left[\begin{array}{c}
u_{1}(z) \\
x_{2}(z)
\end{array}\right] .
$$

The coefficients in Eq. (25) are:

$$
\left\{\begin{array}{l}
a_{0}=k^{2}+2 \omega_{c} k+\omega_{c}^{2}+\hat{\omega}^{2} \\
a_{1}=2 \omega_{c}^{2}+\hat{\omega}^{2}-2 k^{2} \\
a_{2}=k^{2}-2 \omega_{c} k+\omega_{c}^{2}+\hat{\omega}^{2} \\
b_{0}=\omega_{c}^{2}+\omega_{c} k \\
b_{1}=2 \omega_{c}^{2} \\
b_{2}=\omega_{c}^{2}+\omega_{c} k \\
b_{3}=\omega_{c} \hat{\omega} \\
b_{4}=2 \omega_{c} \hat{\omega} \\
b_{5}=\omega_{c} \hat{\omega} \\
k=2 / T_{s}
\end{array} .\right.
$$

These coefficients of the CFM-OSG module can be obtained through simple multiplications and additions when the frequency changes.

A comparison between the proposed method and some improved SOGI-based methods is shown in Table I in terms of the module for positive sequence voltage extraction. The discretization of these methods adopts the Tustin transform. The state variables and the mathematical operations related to the tracking of the grid frequency are not included in the table since they can be the same for all of the different methods.

It can be seen from Table I that with the advantages of low complexity, strong adaptability and high-frequency attenuation, the proposed CFM-OSG-based PLL is an effective way to extract the fundamental positive sequence voltage for digital controllers.

\section{EXPERIMENTS}

In this section, the proposed design procedure has been evaluated through experimental tests in two dSPACE 1103 systems from dSPACE GmbH (Paderborn, GER). A dSPACE 1103 is programed to output signals that simulate the power grid voltage. The other one is used to sample and execute the proposed PLL algorithm. The sampling frequency is fixed at $20 \mathrm{kHz}$, and the nominal frequency is set to $50 \mathrm{~Hz}$. The complex filter parameter and the PI controller parameters are set as $\omega_{c}=260 \mathrm{rad} / \mathrm{s}, k p=0.7$ and $k i=2$.

The performance of the PLL system presented in this paper is experimentally validated under three instances of unbalanced input voltage, distorted input voltage and frequency variation. A TDS2014B oscilloscope (Tektronix, Inc., Oregon, USA) is used as the experimental measuring instrument. In addition, the test items include a three-phase power grid voltage, $u_{\alpha}$ and $u_{\beta}$, $u_{\alpha}+$ and $u_{\beta}+$ (i.e., the fundamental positive sequence components after decoupling), and the frequency and phase of the fundamental positive sequence voltage. Among these, the intermediate variables are sent to the dSPACE D/A converter, and then displayed by the digital oscilloscope.

\section{A. Unbalanced Input Voltage Experiments}

To simulate the grid, a single-phase voltage sag fault is selected as an unbalanced input voltage. Set the symmetrical three-phase voltage as $u_{a}=1 \angle 0^{\circ}$ pu before the fault. The $C$-phase voltage sag fault experimental waveforms are shown in Fig. 9.

When the $C$-phase voltage sag fault emerges, the PLL output frequency fluctuates slightly and then goes back to a stable state after one fundamental cycle. From Fig. 9(c), it is obvious that $u_{\alpha}$ and $u_{\beta}$ are asymmetric. However, the positive sequence components after the decoupling calculation are symmetric. The calculation results show that the fundamental positive sequence voltage in the $\alpha-\beta$ reference frame is $u+=1.22 \angle 0^{\circ} \mathrm{pu}$ before the fault, and $u+=0.82 \angle 0^{\circ} \mathrm{pu}$ after the fault. These results are consistent with the experimental results. 
TABLE I

THE COMPARISON BETWEEN DIFFERENT METHODS

\begin{tabular}{|c|c|c|c|c|c|c|c|}
\hline \multirow[b]{2}{*}{ PLL method } & \multicolumn{4}{|c|}{ General characteristics } & \multicolumn{3}{|c|}{ System complexity } \\
\hline & $\begin{array}{c}\text { Adaptability } \\
\text { under distorted } \\
\text { conditions }\end{array}$ & $\begin{array}{l}\text { Adaptability } \\
\text { under } \\
\text { unbalanced } \\
\text { conditions }\end{array}$ & $\begin{array}{l}\text { Order of input } \\
\text { signal } \\
\text { processing } \\
\text { module }\end{array}$ & $\begin{array}{c}\text { High } \\
\text { frequency } \\
\text { characteristics }\end{array}$ & $\begin{array}{l}\text { Variables } \\
\text { number of } \\
\text { signal } \\
\text { processing } \\
\text { module }\end{array}$ & $\begin{array}{c}\text { Additions } \\
\text { number of } \\
\text { signal } \\
\text { processing } \\
\text { module }\end{array}$ & $\begin{array}{c}\text { Multiplications } \\
\text { number of } \\
\text { signal } \\
\text { processing } \\
\text { module }\end{array}$ \\
\hline $\begin{array}{c}\text { Conventional } \\
\text { SOGI-based } \\
\text { PLL }\end{array}$ & adaptive & maladaptive & 2 & -20dB/decade & 9 & 12 & 16 \\
\hline $\begin{array}{l}\text { DSOGI-based } \\
\text { PLL [33] }\end{array}$ & adaptive & adaptive & 2 & -20dB/decade & 18 & 28 & 34 \\
\hline $\begin{array}{l}\text { Adaptive } \\
\text { notch } \\
\text { filter-based } \\
\text { PLL [34] }\end{array}$ & $\begin{array}{l}\text { Depend on the } \\
\text { selection of } \\
\text { adaptive notch } \\
\text { filters }\end{array}$ & maladaptive & 2 & -20dB/decade & $\begin{array}{c}3+6 n \\
\text { (n denotes the } \\
\text { number of } \\
\text { adaptive notch } \\
\text { filters) }\end{array}$ & $13 n$ & $17 \mathrm{n}$ \\
\hline $\begin{array}{c}\text { Multiple } \\
\text { SOGI-based } \\
\text { PLL [32] }\end{array}$ & $\begin{array}{l}\text { Depend on the } \\
\text { selection of } \\
\text { DSOGI- OSGs }\end{array}$ & adaptive & 2 & -20dB/decade & $\begin{array}{c}6+12 \mathrm{n} \\
\text { (n denotes the } \\
\text { number of } \\
\text { DSOGI- } \\
\text { OSGs) }\end{array}$ & $32 n$ & $34 n-2$ \\
\hline $\begin{array}{l}\text { CFM-OSG- } \\
\text { based PLL }\end{array}$ & adaptive & adaptive & 4 & -40dB/decade & 12 & 37 & 51 \\
\hline
\end{tabular}

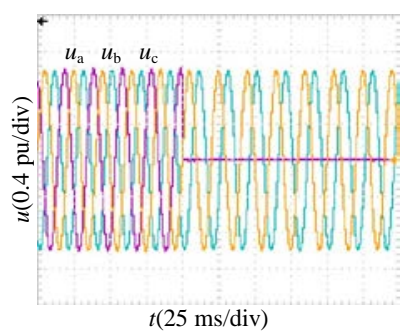

(a)

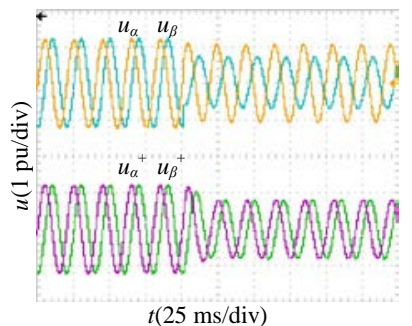

(c)

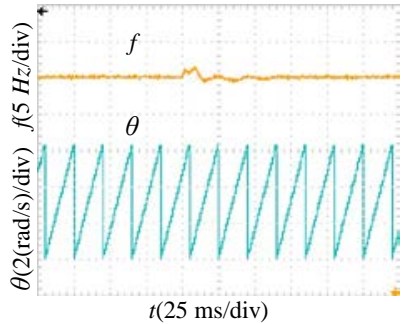

(e)

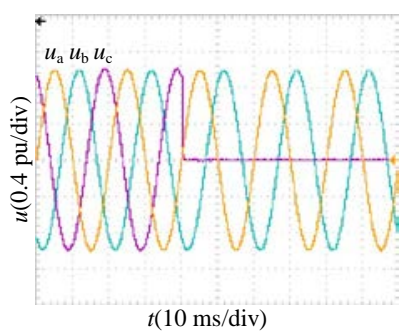

(b)

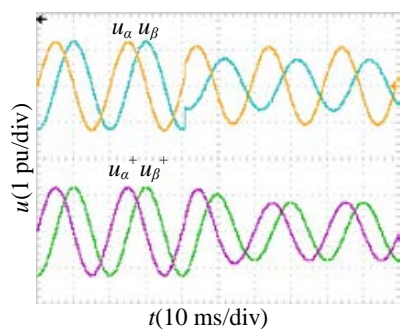

(d)

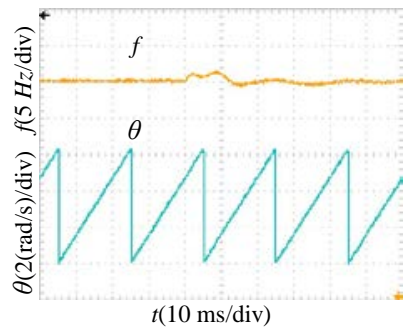

(f)

Fig. 9. System experimental waveforms with single-phase voltage sag fault. (a) Three-phase voltage. (b) Enlarged view of (a). (c) Voltage on the $\alpha-\beta$ reference frame. (d) Enlarged view of (c). (e) Frequency and phase. (f) Enlarged view of (e).

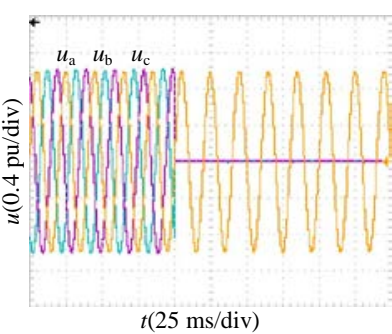

(a)

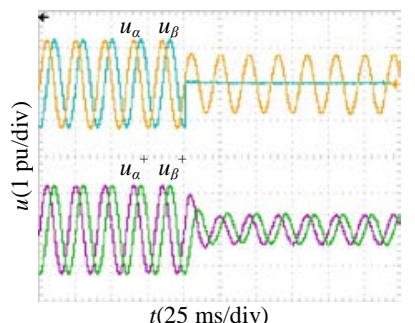

(c)

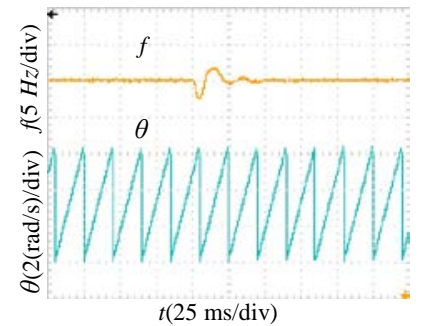

(e)

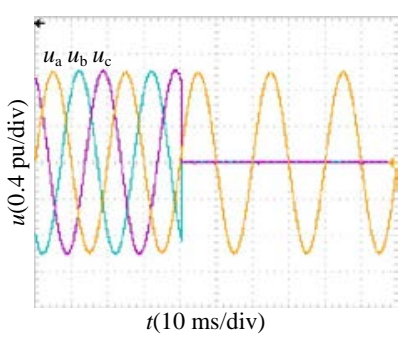

(b)

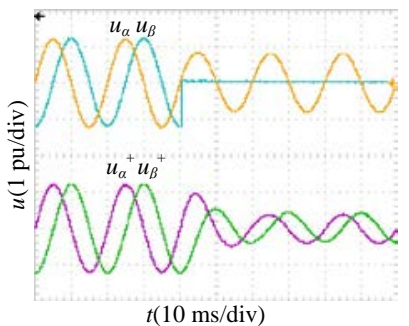

(d)

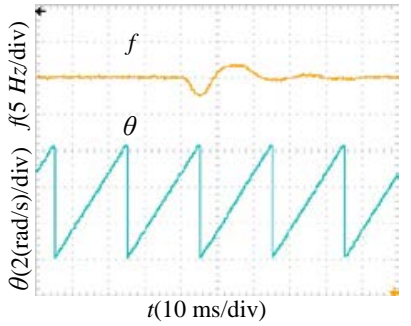

(f)
Fig. 10. System experimental waveforms with two-phase voltage sag fault. (a) Three-phase voltage. (b) Enlarged view of (a). (c) Voltage on the $\alpha-\beta$ reference frame. (d) Enlarged view of (c). (e) Frequency and phase. (f) Enlarged view of (e). 


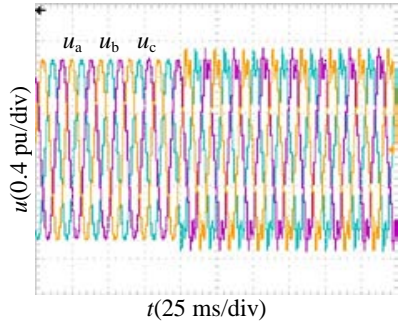

(a)

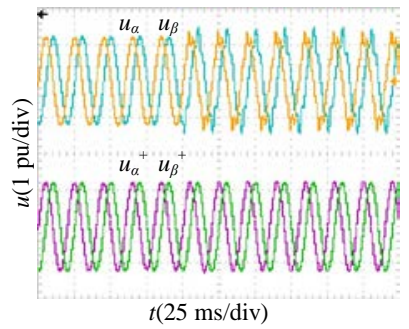

(c)

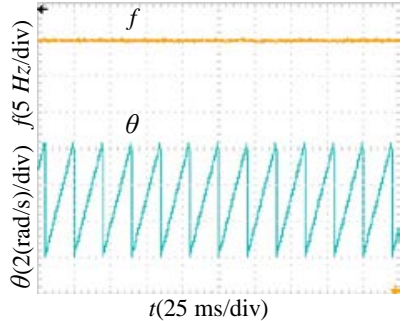

(e)

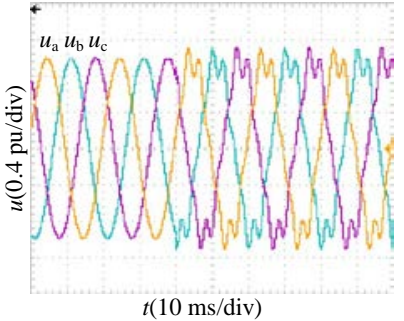

(b)

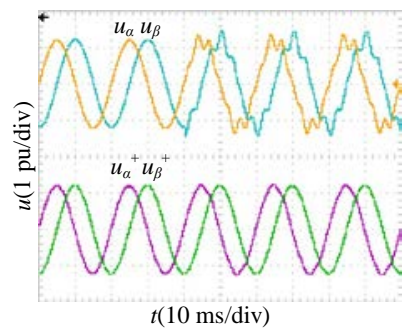

(d)

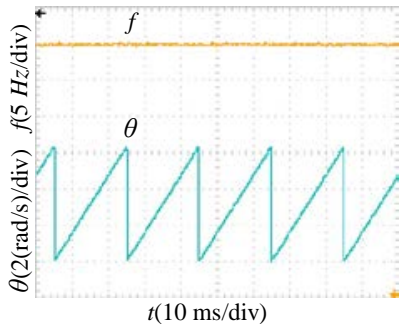

(f)

Fig. 11. System experimental waveforms with distorted input voltage. (a) Three-phase voltage. (b) Enlarged view of (a). (c) Voltage on the $\alpha-\beta$ reference frame. (d) Enlarged view of (c). (e) Frequency and phase. (f) Enlarged view of (e).

Similarly, when the two-phase voltages sag fault emerges, the positive sequence components can still be obtained accurately. The PLL system works steadily, as shown in Fig. 10.

Therefore, the experimental results indicate that the PLL proposed in this paper is effective under the condition of an unbalanced input voltage.

\section{B. Distorted Input Voltage Experiments}

The symmetric fifth and seventh harmonics, the amplitudes of which are $10 \%$ of the fundamental voltage amplitude, are superimposed on the fundamental. The experimental waveforms are shown in Fig. 11. It can be seen that the frequency and phase barely change after the voltage distortion. In addition, the PLL works well even in the case of voltage distortions.

In order to test the working performance of the PLL under unbalanced harmonics conditions, the same fifth harmonic is superimposed on the $B$-phase voltage, and the same seventh harmonic is superimposed on the $C$-phase voltage. The experimental waveforms are shown in Fig. 12. It can be seen from the experimental results that the PLL works well under unbalanced distorted conditions.

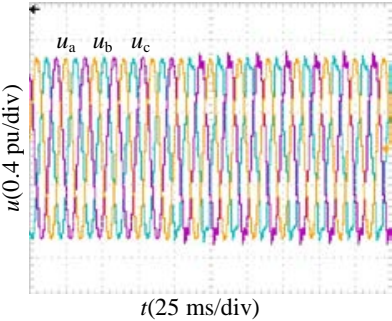

(a)

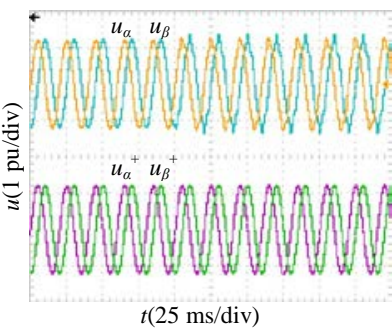

(c)

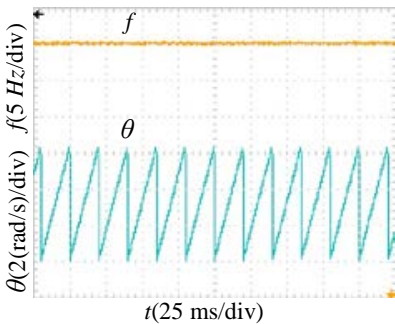

(e)

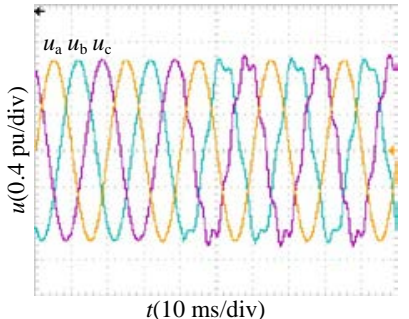

(b)

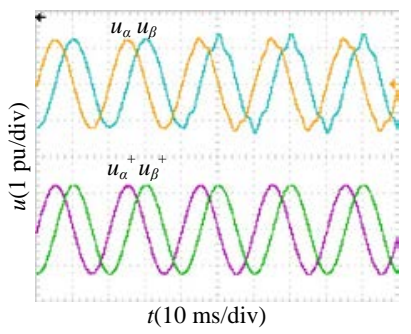

(d)

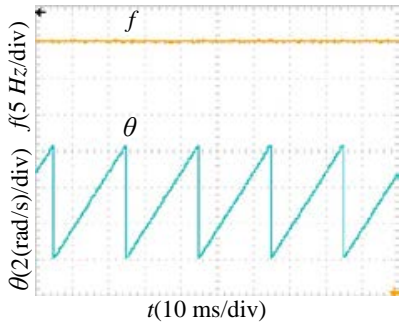

(f)
Fig. 12. System experimental waveforms with unbalanced distorted input voltage. (a) Three-phase voltage. (b) Enlarged view of (a). (c) Voltage on the $\alpha-\beta$ reference frame. (d) Enlarged view of (c). (e) Frequency and phase. (f) Enlarged view of (e).

\section{Frequency Variation Experiment}

Fig. 13 shows the experimental waveforms of unbalanced input voltage frequency variations. The asymmetric input fundamental voltage is set as $u_{a}=1 \angle 0^{\circ} \mathrm{pu}, u_{b}=0.9 \angle-120^{\circ} \mathrm{pu}$, and $u_{c}=0.8 \angle 120^{\circ} \mathrm{pu}$. $f^{*}$ and $f$ denote the real frequency of the input signal and the PLL output frequency, respectively. In addition, $f^{*}$ jumps from $50 \mathrm{~Hz}$ to $47 \mathrm{~Hz}$. It can be observed in the experimental waveforms that the amplitude and phase of the positive sequence component can be detected accurately by the PLL algorithm. In addition, the dynamic response time is less than 2 cycles of the fundamental frequency.

The experimental results indicate that the proposed PLL presents a fast transient response, a high disturbance rejection capability, and a robust performance. All of these show that it is a suitable solution for application in the real-time controllers of power converters connected to the grid.

\section{CONCLUSION}

A new algorithm for use in the grid synchronization of power converters for three-phase systems under unbalanced and distorted operating conditions has been introduced in this 


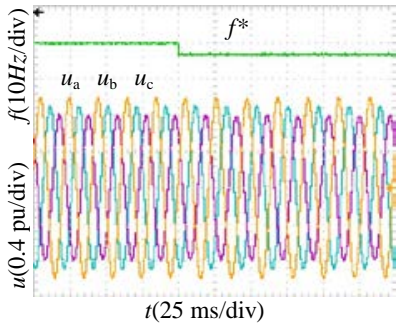

(a)

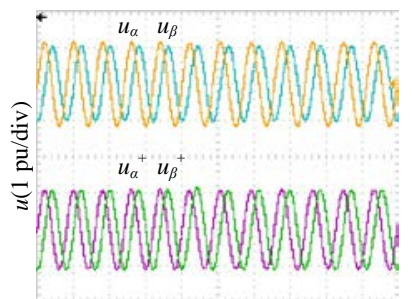

$t(25 \mathrm{~ms} / \mathrm{div})$

(c)

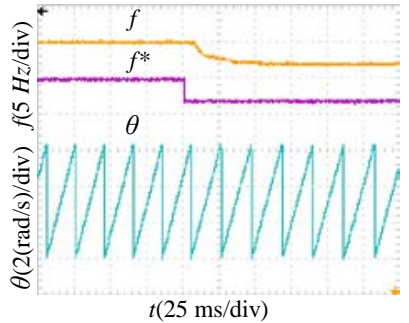

(e)

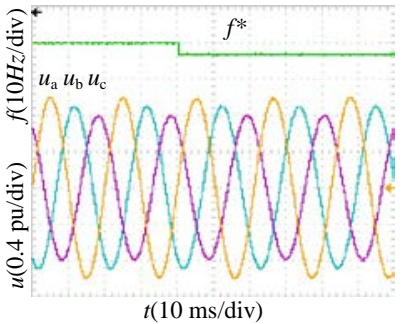

(b)

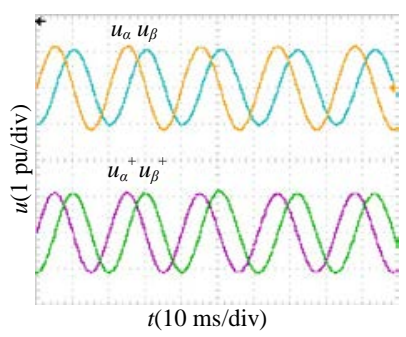

(d)

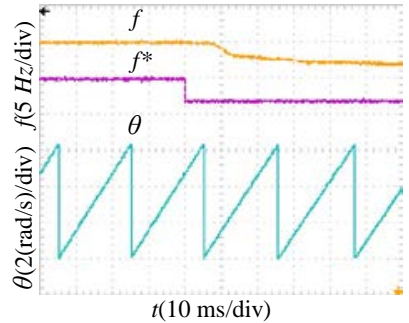

(f)
Fig. 13. System experimental waveforms with frequency variation. (a) Three-phase voltage. (b) Enlarged view of (a). (c) Voltage on the $\alpha-\beta$ reference frame. (d) Enlarged view of (c). (e) Frequency and phase. (f) Enlarged view of (e).

paper. Firstly, first-order complex filters are introduced and their spectral characteristics are analyzed. Then, according to Lyon's method, the relation matrixes of the positive and negative sequence voltage components are derived in the $\alpha-\beta$ reference frame. With the first-order complex filters and the relation matrixes combined, complex filter matrixes are proposed in this paper. Next the complex filter matrixes OSG module is designed through output feedback to extract specific frequency orthogonal signals. Meanwhile, by sending orthogonal signals of the fundamental voltage to the input, a crossing feedback method is presented to decouple the fundamental voltage from the $\alpha$-axis component and the $\beta$-axis component. The parameters and discretized system are then designed for the proposed CFM-OSG PLL. Finally, the theoretical evaluations are verified through experimental studies.

\section{ACKNOWLEDGMENT}

This work is supported by the National Natural Science Foundation of China (Grant No. 51107143) and the Jiangsu Provincial Natural Science Foundation of China (Grant No. BK20130187).

\section{REFERENCES}

[1] F. Blaabjerg, R. Teodorescu, M. Liserre, and A. V. Timbus, "Overview of control and grid synchronization for distributed power generation systems," IEEE Trans. Ind. Electron., Vol. 53, No. 5, pp. 1398-1409, Oct. 2006.

[2] J. W. Choi, Y. K. Kim, and H. G. Kim, "Digital PLL control for single phase photovoltaic system," in Proc. Inst. Elect. Eng. Elect. Power Appl., Vol. 153, No. 1, pp. 40-46, Jan. 2006.

[3] M. Tsili and S. Papathanassiou, "A review of grid code technical requirements for wind farms," IET Renew. Power Gener., Vol. 3, No. 3, pp. 308-332, Sep. 2009.

[4] O. Vainio and S. J. Ovaska, "Noise reduction in zero crossing detection by predictive digital filtering," IEEE Trans. Ind. Electron., Vol. 42, No. 1, pp. 58-62, Feb. 1995.

[5] O. Vainio, S. J. Ovaska, and M. Polla, “Adaptive filtering using multiplicative general parameters for zero-crossing detection," IEEE Tran. Ind. Electron., Vol. 50, No. 6, pp. 1340-1342, Dec. 2003.

[6] M. Karimi-Ghartemani and M. R. Iravani, “A method for synchronous of power electronic converters in polluted and variable frequency environments," IEEE Trans. Power Electron., Vol. 19, No. 3, pp. 1263-1270, Aug. 2004.

[7] A. Routray, A. K. Pradhan, and K. P. Rao, “A novel Kalman filter for frequency estimation of distorted signals in power systems," IEEE Trans. Instrum. Meas., Vol. 51, No. 3, pp. 469-479, Jun. 2002.

[8] H. K. Kwok and D. L. Jones, "Improved instantaneous frequency estimation using an adaptive short-time Fourier transform," IEEE Trans. Signal Process, Vol. 48, No. 10, pp. 2964-2972, Oct. 2000.

[9] B. P. McGrath, D. G. Holmes, and J. J. H. Galloway, "Power converter line synchronization using a discrete Fourier transform (DFT) based on a variable sample rate," IEEE Trans. Power Electron., Vol. 20, No. 4, pp. 877-884, Jul. 2005.

[10] J. P. Costas, “Synchronous communications," in Proc. IEEE IRE, pp. 1713-1718, 1956.

[11] S. Chung, "A phase tracking system for three phase utility interface inverters,” IEEE Trans. Power Electron., Vol. 15, No. 3, pp. 431-438, May 2000.

[12] M. A. Perez, J. R. Espinoza, L. A. Moran, M. A. Torres, and E. A. Araya, "A robust phase-locked loop algorithm to synchronize static-power converters with polluted AC systems,” IEEE Trans. Power Electron., Vol. 55, No. 5, pp. 2185-2192, May 2008.

[13] P. Li, L. Xue, P. Hazucha, T. Karnik, and R. Bashirullah, "A delaylocked loop synchronization scheme for high-frequency multiphase hysteretic DC-DC converters," IEEE J. Solid-State Circuits, Vol. 44, No. 11, pp. 3131-3145, Nov. 2009.

[14] L. L. Lai, C. T. Tse, W. L. Chan, and A. T. P. So, "Real-time frequency and harmonic evaluation using artificial neural networks," IEEE Trans. Power Del., Vol. 14, No. 1, pp. 52-59, Jan. 1999.

[15] H. S. Song, N. Kwanghee, and P. Mutschler, "Very fast phase angle estimation algorithm for a single-phase system having sudden phase angle jumps," in 37th Conf. Rec. IEEE IAS Annu. Meeting, Vol. 2, pp. 925-931, Oct. 2002.

[16] D. Yazdani, A. Bakhshai, G. Joos, and M. Mojiri, “A nonlinear adaptive synchronization technique for grid-connected distributed energy," IEEE Trans. Power Electron., Vol. 23, No. 4, pp. 2181-2186, Jul. 2008.

[17] P. R. M. Santos Filho, P. F. Seixas, P. C. Cortizo, L. Torres, 
and A. F. Souza, "Comparison of three single-phase PLL algorithms for UPS applications,” IEEE Trans. Ind. Electron., Vol. 55, No. 8, pp. 2923-2932, Aug. 2008.

[18] F. D. Freijedo, J. Doval-Gandoy, O. López, and E. Acha, "Tuning of phase-locked loops for power converters under distorted utility conditions,” IEEE Trans. Ind. Appl., Vol. 45, No. 6, pp. 2039-2047, Nov./Dec. 2009.

[19] F. D. Freijedo, A.G. Yepes, O. López, A. Vidal, and J. Doval-Gandoy, “Three-phase PLLs with fast postfault retracking and steady-state rejection of voltage unbalance and harmonics by means of lead compensation," IEEE Trans. Ind. Appl., Vol. 26, No. 1, pp. 85-95, Jan. 2011.

[20] H. E. P. de Souza, F. Bradaschia, F. A. S. Neves, M. C. Cavalcanti, G. M. S. Azevedo, and J.P. de Arruda, “A method for extracting the fundamental-frequency positive-sequence voltage vector based on simple mathematical transformations," IEEE Trans. Ind. Electron., Vol. 56, No. 5, pp. 1539-1547, May 2009.

[21] P. Rodriguez, J. Pou, J. Bergas, J. I. Candela, R. P. Burgos, and D. Boroyevich, "Decoupled double synchronous reference frame PLL for power converters control," IEEE Trans. Power Electron., Vol. 22, No. 2, pp. 584-592, Mar. 2007.

[22] R. Zhang, M. Cardinal, P. Szczesny, and M. Dame, “A grid simulator with control of single-phase power converters in D-Q rotating frame," in Proc. IEEE Power Electron. Spec. Conf., pp. 1431-1436, 2002.

[23] J. Svensson, M. Bongiorno, and A. Sannino,"Practical implementation of delayed signal cancellation method for phase-sequence separation,” IEEE Trans. Power Del., Vol. 22, No. 1, pp. 18-26, Jan. 2007.

[24] M. Bongiorno, J. Svensson, and A. Sannino, "Effect of sampling frequency and harmonics on delay-based phase-sequence estimation method," IEEE Trans. Power Del., Vol. 23, No. 3, pp. 1664-1672, Jul. 2008.

[25] Z. Yao, "Fundamental phasor calculation with short delay," IEEE Trans. Power Del., Vol. 23, No. 3, pp. 1280-1287, Jul. 2008.

[26] R. A. Flores, I. Y. H. Gu, and M. H. J. Bollen, "Positive and negative sequence estimation for unbalanced voltage dips," in Proc. IEEE PES-GM, pp. 2498-2502, 2003.

[27] K. De Brabandere, T. Loix, K. Engelen, B. Bolsens, J. Van den Keybus, J. Driesen, and R. Belmans, "Design and operation of a phase-locked loop with Kalman estimator-based filter for single-phase applications," in Proc. IEEE IECON, pp. 525-530, 2006.

[28] D. Yazdani, M. Mojiri, A. Bakhshai, and G. Joos, “A fast and accurate synchronization technique for extraction of symmetrical components," IEEE Trans. Power Electron., Vol. 24, No. 3, pp. 674-684, Mar. 2009.

[29] P. Rodríguez, A. Luna, R. S. Muñoz-Aguilar, I. Etxeberria-Otadui, R. Teodorescu, and F. Blaabjerg, “A stationary reference frame grid synchronization system for three-phase grid-connected power converters under advese grid conditions,” IEEE Trans. Power Electron., Vol. 27, No. 1, pp. 99-112, Jan. 2012.

[30] S. Golestan, M. Monfared, F.D. Freijedo, and J.M. Guerrero, "Dynamics assessment of advanced single-phase PLL structures,” IEEE Trans. Ind. Electron., Vol. 60, No. 6, pp. 2167-2177, Jun. 2013.

[31] J. A. Suul, A. Luna, P. Rodriguez, and T. Undeland, "Voltage-sensor-less synchronization to unbalanced grids by frequency-adaptive virtual flux estimation," IEEE Trans. Ind. Electron., Vol. 59, No. 7, pp. 2910-2923, Jul. 2012.

[32] P. Rodríguez, A. Luna, I. Candela, R. Mujal, R.
Teodorescu, and F. Blaabjerg, "Multiresonant frequency-locked loop for grid synchronization of power converters under distorted grid conditions," IEEE Trans. Ind. Electron., Vol. 58, No. 1, pp. 127-138, Jan. 2011.

[33] P. Rodríguez, A. Luna, M. Ciobotaru, R. Teodorescu, and F. Blaabjerg,"Advanced grid synchronization system for power converters under unbalanced and distorted operating conditions," in Proc. 32nd Annu. Conf. IEEE Ind. Electron., Nov. 2006, pp. 5173-5178.

[34] D. Yazdani, A. Bakhshai, and P. K. Jain, “A three-phase adaptive notch filter-based approach to harmonic/reactive current extraction and harmonic decomposition," IEEE Trans. Power Electron., Vol. 25, No. 4, pp. 914-923, Apr. 2010.

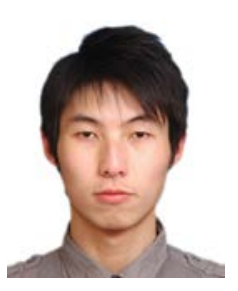

Long-Yue Yang was born in Anhui Province, China, in 1988. He received his B.S. degree in Electrical Engineering and Automation from China University of Mining and Technology (CUMT), Xuzhou, China, in 2010. He is currently working toward his Ph.D. degree in Electrical Engineering in the School of Information and Electrical Engineering, CUMT. His current research interests include power electronics, electric energy quality, and nonlinear control theory.

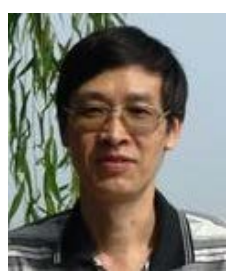

Chong-Lin Wang was born in Anhui Province, China, in 1956. He received his B.S. degree in Electrical Engineering and Automation, and his M.S. and Ph.D. degrees in Electrical Engineering from China University of Mining and Technology (CUMT), Xuzhou, China, in 1981, 1989 and 1997, respectively. He has been a Professor in the School of Information and Electrical Engineering, CUMT, since 1999. He has also been the Chief Professor of the Electrical Safety and Intelligent Electrical Equipment Research Institute, CUMT, since 1999. His current research interests include arc suppression coils and electrical safety.

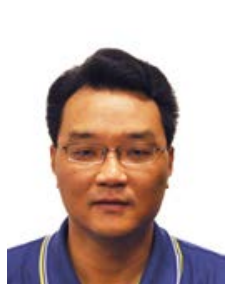

Jian-Hua Liu was born in Henan Province, China, in 1973. He received his B.S. degree in Electrical Engineering and Automation, and his Ph.D. degree in Electrical Engineering from China University of Mining and Technology (CUMT), Xuzhou, China, in 1997 and 2008, respectively. He has been with the School of Information and Electrical Engineering, CUMT, since 1999. He is currently an Associate Professor and a Master Tutor. His current research interests include smart grids and electrical safety.

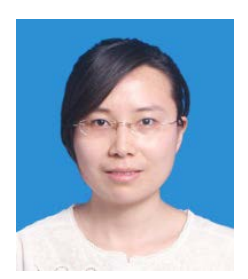

Chen-Xi Jia was born in Anhui Province, China, in 1987. She received her B.S. degree in Electrical Engineering and Automation, and her Ph.D. degree in Electrical Engineering from China University of Mining and Technology (CUMT), Xuzhou, China, in 2009 and 2014, respectively. She has been with the School of Electrical Engineering and Automation, Jiangsu Normal University, Xuzhou, China, since 2014. She is currently presently a Lecturer. Her current research interests include arc suppression coils and electrical safety. 